\title{
The UM/CTIO Magellanic Cloud Emission-line Survey
}

\author{
R. Chris Smith ${ }^{1,2}$ \\ Cerro Tololo Inter-American Observatory, NOAO, Casilla 603, La \\ Serena, Chile
}

and the MCELS team ${ }^{3}$

\begin{abstract}
The Magellanic Clouds are unique in providing sites to study the interstellar medium (ISM) and its components at all scales. To promote the pursuit of such studies, we have begun the Magellanic Cloud Emission-line Survey (MCELS), a deep imaging survey of both of these nearby galaxies in the emission of $\mathrm{H} \alpha$, [S II], and [O III]. The emissionline images will be used in detailed optical and multiwavelength studies of $\mathrm{H}$ II regions, supernova remnants, planetary nebulae, superbubbles, and supergiant shells. Together with parallel surveys at other wavelengths, this survey will provide the foundation upon which to build a deeper understanding of the ISM in the Clouds and other galaxies, from small scales $(\sim 1 \mathrm{pc})$ all the way up to global scales. We present a sample of recent and current work using the MCELS dataset.
\end{abstract}

While we can study some $\mathrm{H}$ II regions, supernova remnants (SNRs), planetary nebulae (PNe), and other nebular structures in detail in our own Galaxy, we lack the global perspective to study their general properties, especially in the optical where interstellar absorption prevents observations of all but the relatively local environment. Studies of distant galaxies provide the global view, but are limited in their ability to examine the detailed interactions on the scales of filaments or stars. The Large and Small Magellanic Clouds are unique in providing us with sites to study the interstellar medium (ISM) and its components at all scales. Their proximity and low foreground absorption make them the ideal laboratories both for studies of individual H II regions, SNRs, and superbubbles, and for investigations of global properties using samples of these objects.

To undertake such research, uniform datasets are needed to provide the basis for the identification, classification, and detailed study of the many components of the ISM. Until recently, optical surveys of the ISM in the Magellanic Clouds were limited to photographic studies performed in individual emission lines (e.g., $\mathrm{H} \alpha$ in Davies, Elliott, \& Meaburn 1976, [O III] in Morgan et al.

\footnotetext{
${ }^{1}$ Also Dept. of Astronomy, Univ. of Michigan, Ann Arbor

${ }^{2}$ Dean B. McLaughlin Fellow

${ }^{3}$ MCELS team includes: J. Bregman, Y.-H. Chu, R. Ciardullo, G. Jacoby, R. Kennicutt, S. Oey, F. Winkler, \& D. Zaritsky
} 
1995). More recent surveys with modern CCD detectors have generally been limited either in area or in resolution (e.g., Kennicutt et al. 1995). With the relatively recent combination of a large format CCD detector with the Schmidt telescope at CTIO, we have initiated a deep multiple emission-line CCD survey of both the LMC and SMC, the UM/CTIO Magellanic Cloud Emission-line Survey (MCELS).

\section{MCELS Details}

Taking advantage of the wide field of view available on the UM/CTIO Curtis Schmidt telescope coupled to the sensitivity and large area of newer CCDs, we are imaging the central $8 \times 8 \mathrm{deg}$. of the LMC and the central $3.5 \times 4.5 \mathrm{deg}$. of the SMC, covering most of the gaseous extent of both galaxies. The $2048^{2}$ CCD cameras used provide $3-4^{\prime \prime}$ resolution with a field of view of more than 1 degree $^{2}$. A grid of multiple, overlapping fields has been planned to provide uniform coverage of the Clouds with multiple samples at each point.

In order to isolate the emission lines, we are using narrow-band interference filters of $\mathrm{H} \alpha\left(\lambda_{c}=6563, \Delta \lambda=30 \AA\right)$, [S II] $\left(\lambda_{c}=6724, \Delta \lambda=50 \AA\right)$, and [O III] $\left(\lambda_{c}=5007, \Delta \lambda=40 \AA\right.$ ). Images in two "continuum-band" filters (at $\lambda_{c}=6850$, $\Delta \lambda=95 \AA$ and $\lambda_{c}=5130, \Delta \lambda=155 \AA$ ) are also obtained to allow subtraction of the stellar background, leaving only the emission-line contribution. We plan to reach a limiting surface brightness in all three emission lines of approximately $3 \times 10^{-17} \mathrm{ergs} \mathrm{cm}^{-2} \mathrm{~s}^{-1} \operatorname{arcsec}^{-2}$, a surface brightness roughly equivalent to the diffuse $\mathrm{H} \alpha$ background measured in the LMC by Kennicutt et al. (1995).

\section{Preliminary Survey Results}

MCELS observations began in November 1995, and as of February 1998 we have completed almost three-quarters of the survey of both Clouds. Preliminary analysis of both survey and pre-survey emission-line data is already providing interesting and exciting results. Highlights of these include:

- Newly Identified SNRs: The sample of SNRs in both the LMC and SMC suffers from considerable incompleteness. This incompleteness has been highlighted by the ease with which we have discovered several previously missed SNRs in the LMC. Smith et al. (1994) identified two SNRs in a single $30^{\prime} \times 30^{\prime}$ field with the combination of optical emission-line and X-ray datasets (Figure 1). Since then, we have identified, or provided corroborating data for, five more SNRs (e.g., Chu et al. 1995), along with several more SNR candidates.

- Multiwavelength Studies of Known SNRs: Despite its incompleteness, the Magellanic Cloud sample of SNRs is still the best sample of remnants available for detailed study. Williams et al. have taken the lead on combining our MCELS emission-line data with both radio and X-ray datasets to examine several LMC SNRs in detail, including the colliding remnants DEM316 (Williams et al. 1997) and two examples of SNR blowouts, N11L and N86 (Williams et al. 1999). Tavarez et al. (1999) have also been pursuing such multiwavelength studies, including near-IR information into the analysis. 


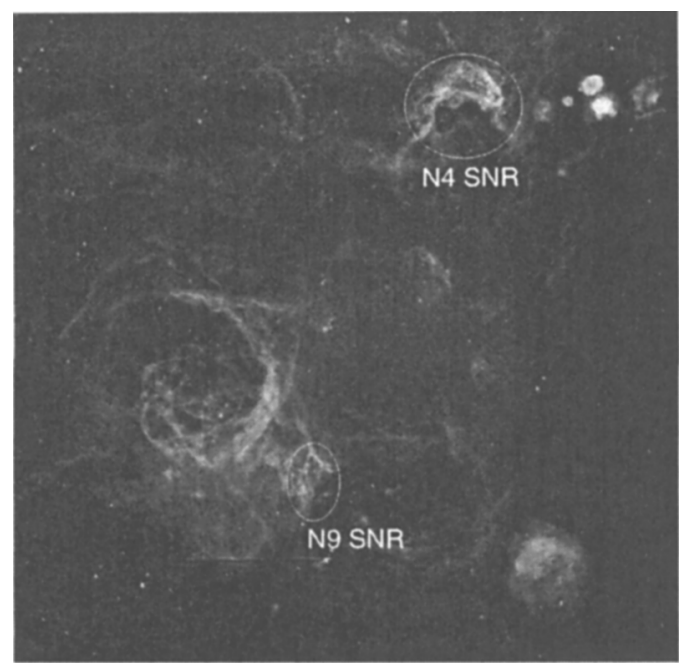

Figure 1. This continuum-subtracted $\mathrm{H} \alpha$ image of a $30^{\prime} \times 30^{\prime}$ region on the western edge of the LMC reveals the rich variety of structures which can be seen in the MCELS data, including the newly identified SNRs in the N4 and N9 HII complexes. The subtraction of stellar light allows even the faintest filaments to be traced out.

- HII Complexes: On the larger scale, our observations have provided input for several studies of HII complexes and superbubbles in the LMC. Mac Low et al. (1998) showed that the N11 superbubble is an intermediate case in the known range of $\mathrm{X}$-ray overluminosity with respect to superbubble models, and Magnier et al. (1996) demonstrated that the filamentary structure to the south of the superbubble N44 (Figure 2) is most likely due to a blowout from the shell. Kim et al. (1998) added H I data to the analysis of N44, showing that the neutral component has as much as seven times the kinetic energy of the ionized component.

- Large-scale Structures: Points et al. (1999) have used MCELS data in their study of the supergiant shell LMC2. Combining the optical emission-line data with the X-ray dataset covering the region, they present a detailed casestudy of LMC2. And at the largest scale, Winkler et al. (1999) have provided a global view of the ISM in the SMC, highlighting the variety of structure and detail which can be seen in the MCELS dataset.

\section{Future}

The work described above is just the beginning of our exploration of the ISM of the Magellanic Clouds using the MCELS observations. When complete, the survey will provide definitive samples of wind-blown bubbles, PNe, and SNRs, as well as calibrated multiple emission-line images of HII regions, superbubbles, and supergiant shells. More information about the MCELS project, as well as 


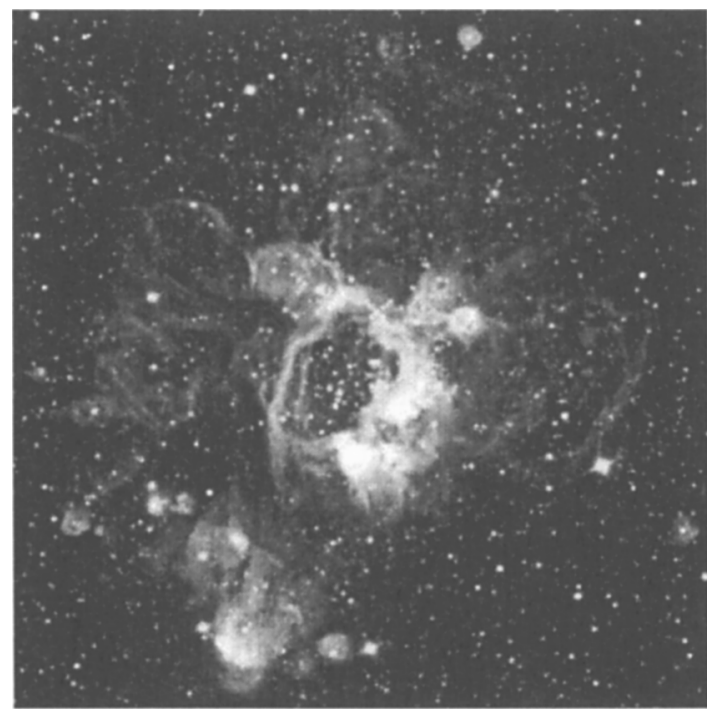

Figure 2. The N44 superbubble in the light of $\mathrm{H} \alpha$.

details about the datasets and their eventual public release, can be found at http://www.astro.lsa.umich.edu/mcels.

Acknowledgments. We gratefully acknowledge the support of CTIO and all of the assistance which has been provided in upgrading the Curtis Schmidt telescope. The MCELS is funded through the generous support of the Dean B. McLaughlin fund at the Univ. of Michigan and through NSF Grant \#9540747.

\section{References}

Chu, Y.-H., et al. 1995, AJ, 109, 1729

Davies, R.D., Elliott, K.H., \& Meaburn, J. 1976, MmRAS, 81, 89

Kennicutt, R.C., et al. 1995, AJ, 109, 594

Kim, S., et al. 1998, ApJ, 503, 729

Mac Low, M.-M., et al. 1998, ApJ, 493, 260

Magnier, E.A., et al. 1996, ApJ, 464, 829

Morgan, D.H., 1995, A\&AS, 112, 445

Points, S., et al. 1999, ApJ, 518, 298

Smith, R.C., et al. 1994, AJ, 108, 1266

Tavarez, M., et al. 1999, in preparation

Williams, R., et al. 1997, ApJ, 480, 618

Williams, R., et al. 1999, ApJ, 514, 798

Winkler, P.F., et al. 1999, this volume 